\title{
Design of parameter-scheduled state-feedback controllers using shifting specifications
}

\author{
Damiano Rotondo ${ }^{\mathrm{a}, *}$, Fatiha Nejjari ${ }^{\mathrm{a}}$, Vicenç Puig ${ }^{\mathrm{a}}$ \\ ${ }^{a}$ Department of Automatic Control (ESAII), Technical University of Catalonia (UPC), Rambla de \\ Sant Nebridi 10, 08222 - Terrassa (Spain). Tel: +34 937398973
}

\begin{abstract}
In this paper, the problem of designing a parameter-scheduled state-feedback controller is investigated. The paper presents an extension of the classical regional pole placement, $\mathscr{H}_{2}$ control and $\mathscr{H}_{\infty}$ control problems, so as to satisfy new specifications, that will be referred to as shifting pole placement control, shifting $\mathscr{H}_{2}$ control and shifting $\mathscr{H}_{\infty}$ control, respectively. By introducing some parameters, or using the existing ones, the controller can be designed in such a way that different values of these parameters imply different regions where the closed-loop poles are situated, or different performances in the $\mathscr{H}_{2}$ or $\mathscr{H}_{\infty}$ sense. The proposed approach is derived within the so-called Lyapunov shaping paradigm, where a single quadratic Lyapunov function is used for ensuring stability and desired performances in spite of arbitrary parameter time variation. The problem is analyzed in the continuous-time LPV case, even though the developed theory could be applied to LTI systems in cases when it is desired to vary the control system performances online. Results obtained in simulation demonstrate the effectiveness and the relevant features of the proposed approach.
\end{abstract}

Keywords: Linear parameter-varying systems, regional pole assignment, multivariable feedback control, $\mathscr{H}_{\infty}$ control, $\mathscr{H}_{2}$ control, linear matrix inequalities.

${ }^{*}$ Corresponding author

Email addresses: damiano.rotondo@yahoo.it (Damiano Rotondo), fatiha.nejjari@upc.edu (Fatiha Nejjari), vicenc.puig@upc.edu (Vicenç Puig) 


\section{Introduction}

In the last decades, linear matrix inequalities (LMIs) have emerged as a powerful formulation and design technique for a variety of linear control problems [1]. Indeed, a wide range of problems arising in systems and control theory can be reduced to convex optimization problems that involve LMIs, that are appealing from a practical standpoint, since they can be solved by efficient algorithms. Among the problems that lead to LMIs, there are: regional pole placement, $\mathscr{H}_{\infty}$ performance and $\mathscr{H}_{2}$ performance [2].

The design of control laws that place the closed-loop poles of the system to be controlled at some desired location of the complex plane is one of the most relevant problems in control theory. Historically, the problem of exact pole placement, where closed-loop poles are required to lie at some desired location, was studied at the beginning [3]. However, in cases where exact pole placement is not required, regional pole placement, where closed-loop poles have to be placed within a prescribed region of the plane, is sufficient. Remarkable results were obtained in [2], where the problem of designing state- or output- feedback $H_{\infty}$ controllers that satisfy additional constraints on the closed-loop pole location was addressed. Sufficient conditions for feasibility, expressed in terms of LMIs easily tractable from a numerical point of view, were derived for a general class of convex regions of the complex plane. [2] started from an extension of the Lyapunov characterization of stability made in [4], so as to obtain LMI-based conditions for pole clustering, obtained with the introduction of the so-called LMI Regions.

In [5], a parameter-dependent Lyapunov approach has been used to deal with constant or time-varying uncertainty, obtaining less conservative results in the case of slow parametric variations. Further research has aimed to achieve many objectives, among which to enforce robustness [6] or to solve the pole placement problem for filter design [7]. The big amount of articles appeared in the last decade, e.g. $[8,9,10,11,12,13]$, demonstrates that the regional pole placement problem is still a hot topic of investigation.

On the other hand, a lot of research performed in the last decades has involved two performance measures: the $\mathscr{H}_{2}$ and the $\mathscr{H}_{\infty}$ norms, both defined in the frequency-domain for stable transfer matrices. The $\mathscr{H}_{2}$ optimal control theory was heavily studied in the 1960s as the linear quadratic gaussian (LQG) optimal control theory. On the other hand, the $\mathscr{H}_{\infty}$ optimal control theory, introduced in an input-output setting [14], reached a mature state in the late 1980s, when it was completed with state space formulations [15] and comprehensive comparisons with the $\mathscr{H}_{2}$ control problem [16]. 
Necessary and sufficient conditions for the existence of an $\mathscr{H}_{\infty}$ controller of any order were given in terms of LMIs in [17] and [18]. These LMIs correspond to the inequality counterpart of the $\mathscr{H}_{\infty}$ Riccati equations. Additional flexibility in the $\mathscr{H}_{\infty}$ control design has been introduced through the use of parameterdependent quadratic Lyapunov functions in [19]. A systematic design technique that combines the good aspects of both $\mathscr{H}_{2}$ and $\mathscr{H}_{\infty}$ methods has been developed later in [20]. Similar to the regional pole placement case, the $\mathscr{H}_{2}$ and $\mathscr{H}_{\infty}$ control problems have been the subject of recent research, e.g. [21, 22].

Polytopic systems constitute an important field for regional pole placement and $\mathscr{H}_{2} / \mathscr{H}_{\infty}$ application. The polytopic representation is one of the most used approaches for describing uncertainty or variability of the parameters in linear parameter-varying (LPV) systems. Looking at [2, 5, 19, 7, 21, 8, 12, 13], it can be seen that a lot of effort has been put in developing techniques for regional pole placement and $\mathscr{H}_{2} / \mathscr{H}_{\infty}$ control of polytopic systems, taking advantage of some useful properties of LMIs that allow to assure that their satisfaction at some points, i.e. the vertices of the polytope, implies their satisfaction in all points inside the polytope.

In this work, the problem of designing a parameter-scheduled state-feedback controller is investigated. The main novelty and contribution of this paper is to take advantage of the properties of polytopes and LMIs to solve new problems, that can be seen as extensions of the classical regional pole placement control, $\mathscr{H}_{2}$ control and $\mathscr{H}_{\infty}$ control problems, that will be referred to as shifting pole placement control, shifting $\mathscr{H}_{2}$ control and shifting $\mathscr{H}_{\infty}$ control. In these new problems, by introducing some parameters, or using the existing ones, the controller can be designed in such a way that different values of these parameters imply different regions where the closed-loop poles are situated, or different performances in the $\mathscr{H}_{2}$ or $\mathscr{H}_{\infty}$ sense. This paper constitutes an extension of [23], where only the case of shifting pole placement control was considered.

From a practical point of view, reasons for which such a problem can be of interest include all situations where some performance degradation could be desirable, e.g. high/low-gain control, control of systems with saturation non-linearities [24], graceful performance degradation for active fault-tolerant control [25] and actuator health degradation avoidance [26].

As in [27], the proposed approach is derived within the so-called Lyapunov shaping paradigm, where a single quadratic Lyapunov function is used for ensuring stability and desired performances in spite of arbitrary parameter time variation, and consequently the obtained results are in some way conservative. Other types of Lyapunov functions could be considered in order to reduce such con- 
servativeness, e.g. parameter-dependent [28, 19] or piecewise [29] ones, but the application of such functions goes beyond the goal of this paper. The problem is analyzed in the case of continuous-time LPV systems, even though the developed theory could be applied to LTI systems in cases when it is desired to vary the control system performances online. It should be stated that the multiple model (MM) [30] or the fuzzy Takagi-Sugeno (TS) [31, 32, 33] paradigms could be considered instead of the LPV one. According to [34, 35, 36], these paradigms are very similar, thus the results obtained in this work could be easily extended to MM and TS systems. Results obtained in simulation are presented, demonstrating the effectiveness and the relevant features of the proposed approach.

The paper is organized as follows: in Section 2, the notation used throughout the paper is explained, along with some definitions and known results that are a starting point for the rest of the paper. In Section 3, the approach for the design of parameter-scheduled state-feedback controller using shifting specifications, that is the main topic of this paper, is outlined for the LPV case. In Section 4, an example is used to show an application of the proposed approach. Finally, conclusions are outlined in Section 5.

\section{Background}

For real symmetric matrices $M$, the notation $M>0$ stands for positive definite and indicates that all the eigenvalues of $M$ are positive. Similarly, $M<0$ means negative definite, that is, all the eigenvalues of $M$ are negative. The symbol $\otimes$ denotes the Kronecker product of matrices [37], and the notation $M=\left[m_{k l}\right]_{1 \leq k, l \leq m}$ means that $M$ is an $m \times m$ matrix with generic entry $m_{k l}$. Finally, a matrix polytope is defined as the convex hull of a finite number of matrices $H_{i}$ with the same dimensions:

$$
\operatorname{Co}\left\{H_{i}, i=1, \ldots, N\right\}=\left\{\sum_{i=1}^{N} \alpha_{i}(\theta) H_{i}, \alpha_{i}(\theta) \geq 0, \sum_{i=1}^{N} \alpha_{i}(\theta)=1\right\}
$$

Let $\mathscr{D}$ be a subregion of the complex left half plane. A continuous-time dynamical system $\dot{x}(t)=A x(t)$ is called $\mathscr{D}$-stable if all its poles lie in $\mathscr{D}$, that is, all the eigenvalues of the matrix $A$ lie in $\mathscr{D}$ (then, the matrix $A$ is called $\mathscr{D}$-stable). In [2], a class of regions suitable for LMI-based synthesis is defined as follows:

Definition 1. [2] A subset $\mathscr{D}$ of the complex plane is called an LMI region if there exist a symmetric matrix $\alpha=\left[\alpha_{k l}\right] \in \mathbb{R}^{m \times m}$ and a matrix $\beta=\left[\beta_{k l}\right] \in \mathbb{R}^{m \times m}$ such 
that:

$$
\mathscr{D}=\left\{z \in \mathbb{C}: f_{\mathscr{D}}(z)<0\right\}
$$

with the characteristic function $f_{\mathscr{D}}(z)$ defined as:

$$
f_{\mathscr{D}}(z)=\alpha+z \beta+\bar{z} \beta^{T}=\left[\alpha_{k l}+\beta_{k l} z+\beta_{l k} \bar{z}\right]_{1 \leqslant k, l \leqslant m}
$$

where $\bar{z}$ is the complex conjugate of $z$.

In other words, an LMI region is a convex subset of the complex plane that is representable by an LMI in $z$ and $\bar{z}$. In [2], it is shown that LMI regions not only include a wide variety of typical clustering regions, but also form a subset of the convex regions that are symmetric with respect to the real axis, covering most practical needs for control purposes. Among the regions that are representable as LMI regions, there are:

- Left-hand semiplanes $\operatorname{Re}(z)<\lambda$

$$
\alpha=-2 \lambda \quad \beta=1
$$

- Right-hand semiplanes $\operatorname{Re}(z)>\lambda$

$$
\alpha=2 \lambda \quad \beta=-1
$$

- Disks of radius $r$ and center $(-q, 0)$

$$
\alpha=\left(\begin{array}{cc}
-r & q \\
q & -r
\end{array}\right) \quad \beta=\left(\begin{array}{ll}
0 & 1 \\
0 & 0
\end{array}\right)
$$

- Horizontal strips $-\omega<\operatorname{Im}(z)<\omega$

$$
\alpha=\left(\begin{array}{cc}
-2 \omega & 0 \\
0 & -2 \omega
\end{array}\right) \quad \beta=\left(\begin{array}{cc}
0 & 1 \\
-1 & 0
\end{array}\right)
$$

Pole location in a given LMI region can be characterized in terms of the $m \times m$ matrix:

$M_{\mathscr{D}}(A, X)=\alpha \otimes X+\beta \otimes(A X)+\beta^{T} \otimes(A X)^{T}=\left[\alpha_{k l} X+\beta_{k l} A X+\beta_{l k} X A^{T}\right]_{1 \leq k, l \leq m}$

through the following theorem: 
Theorem 1. [2] The matrix $A$ is $\mathscr{D}$-stable if and only if there exists a positive definite symmetric matrix $X=X^{T}>0$ such that:

$$
M_{\mathscr{D}}(A, X)<0
$$

Proof: See [2].

$M_{\mathscr{D}}$ in (4) and $f_{\mathscr{D}}(z)$ in (3) are related by the substitution $\left(X, A X, X A^{T}\right) \leftrightarrow$ $(1, z, \bar{z})$.

The $\mathscr{H}_{\infty}$ norm measures the system input-output gain for finite energy signals. A constraint on the $\mathscr{H}_{\infty}$ norm can be interpreted as a disturbance rejection performance. It is defined as follows:

Definition 2. [38] For a continuous-time stable real-rational transfer matrix $P(s)$, the $\mathscr{H}_{\infty}$ norm is defined as:

$$
\|P(s)\|_{\infty}=\sup _{\omega \in \mathbb{R}} \sigma_{\max }(P(j \omega))
$$

where $\sigma_{\max }(M)$ stands for the largest singular value of the matrix $M$.

Consider a linear time-invariant (LTI) system described by:

$$
\begin{gathered}
\dot{x}(t)=A x(t)+B_{w} w(t)+B_{u} u(t) \\
z_{\infty}(t)=C_{z_{\infty}} x(t)+D_{z_{\infty} w} w(t)+D_{z_{\infty} u} u(t)
\end{gathered}
$$

where $x(t) \in \mathbb{R}^{n_{x}}$ is the system state, $w(t) \in \mathbb{R}^{n_{w}}$ is a vector of exogenous inputs (such as reference signals, disturbance signals, sensor noise), $u(t) \in \mathbb{R}^{n_{u}}$ is the control input, $z_{\infty}(t) \in \mathbb{R}^{n_{z_{\infty}}}$ is a vector of output signals related to the $\mathscr{H}_{\infty}$ performance of the system, and $A, B_{w}, B_{u}, C_{z_{\infty}}, D_{z_{\infty} w}, D_{z_{\infty} u}$ are matrices of appropriate dimensions. Also consider that the system (7)-(8) is under state-feedback control:

$$
u(t)=K x(t)
$$

and let $T_{z_{\infty} w}(s)$ denote the transfer function from $w$ to $z_{\infty}$, and $\left(A_{c l}, B_{c l}, C_{c l \infty}, D_{c l \infty}\right)$ one of its possible state-space realizations. The $\mathscr{H}_{\infty}$ norm constraint $\left\|T_{z_{\infty} w}(s)\right\|_{\infty}<$ $\gamma$ is equivalent to the existence of a positive-definite symmetric matrix $X_{\infty}=X_{\infty}^{T}>$ 0 such that [2]:

$$
\left(\begin{array}{ccc}
A_{c l} X_{\infty}+X_{\infty} A_{c l}^{T} & B_{c l} & X_{\infty} C_{c l \infty}^{T} \\
B_{c l}^{T} & -I & D_{c l \infty}^{T} \\
C_{c l \infty} X_{\infty} & D_{c l \infty} & -\gamma^{2} I
\end{array}\right)<0
$$


This result is known as bounded real lemma (BRL) [39].

The $\mathscr{H}_{2}$ norm is equal to the root-mean-square of the impulse response of the system. It measures the steady-state covariance (or power) of the output response to a unit white noise input. It is defined as follows:

Definition 3. [40] For a continuous-time stable real-rational transfer matrix $P(s)$, the $\mathscr{H}_{2}$ norm is defined as:

$$
\|P(s)\|_{2}=\sqrt{\frac{1}{2 \pi} \int_{-\infty}^{+\infty} \operatorname{Tr}\left(P(j \omega)^{H} P(j \omega)\right) d \omega}
$$

Consider an LTI system described by (7) and by:

$$
z_{2}(t)=C_{z_{2}} x(t)+D_{z_{2}} u(t)
$$

where $z_{2}(t) \in \mathbb{R}^{n_{z_{2}}}$ is a vector of output signals related to the $\mathscr{H}_{2}$ performance of the system, and $C_{z_{2}}, D_{z_{2} u}$ are matrices of appropriate dimensions. Also consider that the system (7) and (12) is under state-feedback control (9), and let $T_{z_{2} w}(s)$ denote the transfer function from $w$ to $z_{2}$, and $\left(A_{c l}, B_{c l}, C_{c l 2}, D_{c l 2}\right)$ one of its possible state-space realizations. The $\mathscr{H}_{2}$ norm constraint $\left\|T_{z_{2} w}(s)\right\|_{2}<v$ is equivalent to the existence of a positive definite symmetric matrix $X_{2}=X_{2}^{T}>0$ and a symmetric matrix $Y=Y^{T}$ such that [2]:

$$
\begin{gathered}
\operatorname{trace}(Y)<v^{2} \\
\left(\begin{array}{cc}
Y & C_{c l 2} X_{2} \\
X_{2} C_{c l 2}^{T} & X_{2}
\end{array}\right)>0 \\
A_{c l} X_{2}+X_{2} A_{c l}^{T}+B_{c l} B_{c l}^{T}<0
\end{gathered}
$$

Finally, let us recall the definition of linear parameter-varying (LPV) systems. Following the terminology of [41], LPV systems are linear time-varying (LTV) plants whose state-space matrices are fixed functions of some vector of varying parameters $\theta(t) \in \Theta \subset \mathbb{R}^{n_{\theta}}$. For example, the LPV version of a system described by (7), (8) and (12) is the following:

$$
\begin{gathered}
\dot{x}(t)=A(\theta(t)) x(t)+B_{w}(\theta(t)) w(t)+B_{u}(\theta(t)) u(t) \\
z_{\infty}(t)=C_{z_{\infty}}(\theta(t)) x(t)+D_{z_{\infty} w}(\theta(t)) w(t)+D_{z_{\infty} u}(\theta(t)) u(t) \\
z_{2}(t)=C_{z_{2}}(\theta(t)) x(t)+D_{z_{2} u}(\theta(t)) u(t)
\end{gathered}
$$


Definition 4. [27] The LPV system (16)-(18) is called polytopic when it can be represented by state-space matrices whose dependence on the parameter vector $\theta$, that ranges over a polytope, is affine:

$$
\begin{gathered}
\left(\begin{array}{ccc}
A(\theta(t)) & B_{w}(\theta(t)) & B_{u}(\theta(t)) \\
C_{z_{\infty}}(\theta(t)) & D_{z_{\infty} w}(\theta(t)) & D_{z_{\infty} u}(\theta(t)) \\
C_{z_{2}}(\theta(t)) & 0 & D_{z_{2} u}(\theta(t))
\end{array}\right) \\
\quad \in \operatorname{Co}\left\{\left(\begin{array}{ccc}
A_{i} & B_{w, i} & B_{u, i} \\
C_{z_{\infty}, i} & D_{z_{\infty} w, i} & D_{z_{\infty} u, i} \\
C_{z_{2}, i} & 0 & D_{z_{2} u, i}
\end{array}\right), i=1, \ldots, N\right\}
\end{gathered}
$$

\section{State-feedback using shifting specifications}

Consider the LPV system given by (16)-(18), and divide the $n_{\theta}$-dimensional set $\Theta$ into three subsets, i.e. an $n_{\theta_{s}}$-dimensional set $\Theta_{s}$, an $n_{\theta_{r}}$-dimensional set $\Theta_{r}$, and an $n_{\theta_{p}}$-dimensional set $\Theta_{p}$, such that:

$$
\Theta=\Theta_{s} \times \Theta_{r} \times \Theta_{p}
$$

where $\times$ denotes the Cartesian product of sets. Then, the vector of varying parameters $\theta(t)$ can be written as:

$$
\theta(t)=\left[\begin{array}{lll}
\theta_{s}(t) & \theta_{r}(t) & \theta_{p}(t)
\end{array}\right]^{T}
$$

where $\theta_{s}(t)$ are varying parameters used to schedule the controller (they would correspond to variables that can be either measured or estimated), $\theta_{r}(t)$ are parameters that are not used to schedule the controller, and against whose variation robustness must be guaranteed (they would correspond to unmeasurable variables that cannot be estimated, but also to the unknown but bounded uncertainties affecting the system, e.g. the ones arising from noise or estimation errors in the variables $\theta_{s}(t)$ and $\theta_{p}(t)$ [42]), and $\theta_{p}(t)$ are varying parameters used to schedule not only the controller as in the case of $\theta_{s}(t)$, but also the shifting specifications $\mathscr{D}\left(\theta_{p}(t)\right), \gamma\left(\theta_{p}(t)\right)$ and $v\left(\theta_{p}(t)\right)$, defined formally in the following. $\Theta_{s}, \Theta_{r}$ and $\Theta_{p}$ are assumed to be polytopes, such that:

$$
\begin{gathered}
\theta_{S}(t)=\sum_{i=1}^{S} s_{i}\left(\theta_{S}(t)\right) \theta_{s, i} \quad \text { with } \quad \sum_{i=1}^{S} s_{i}\left(\theta_{s}(t)\right)=1 \quad \text { and } \quad s_{i}\left(\theta_{S}(t)\right) \geq 0 \forall i \\
\theta_{r}(t)=\sum_{j=1}^{R} r_{j}\left(\theta_{r}(t)\right) \theta_{r, j} \quad \text { with } \quad \sum_{j=1}^{R} r_{j}\left(\theta_{r}(t)\right)=1 \quad \text { and } \quad r_{j}\left(\theta_{r}(t)\right) \geq 0 \forall j
\end{gathered}
$$




$$
\theta_{p}(t)=\sum_{h=1}^{P} \pi_{h}\left(\theta_{p}(t)\right) \theta_{p, h} \quad \text { with } \quad \sum_{h=1}^{P} \pi_{h}\left(\theta_{p}(t)\right)=1 \quad \text { and } \quad \pi_{h}\left(\theta_{p}(t)\right) \geq 0 \forall h
$$

with $S, R$ and $P$ the number of vertices, denoted by $\theta_{s, i}, \theta_{r, j}$ and $\theta_{p, h}$, of $\Theta_{s}, \Theta_{r}$ and $\Theta_{p}$, respectively. Then, $\Theta$ is a Cartesian product of polytopes [43], such that:

$$
\theta(t)=\sum_{i=1}^{S} s_{i}\left(\theta_{s}(t)\right) \sum_{j=1}^{R} r_{j}\left(\theta_{r}(t)\right) \sum_{h=1}^{P} \pi_{h}\left(\theta_{p}(t)\right) \theta_{i j h}
$$

where $\theta_{i j h}$ is defined as:

$$
\theta_{i j h}=\left[\begin{array}{lll}
\theta_{s, i} & \theta_{r, j} & \theta_{p, h}
\end{array}\right]^{T}
$$

In the following, the problem of designing the controller:

$$
u(t)=K\left(\theta_{s}(t), \theta_{p}(t)\right) x(t)
$$

so as to satisfy one of the following specifications:

- shifting pole placement

- shifting bound on the $\mathscr{H}_{\infty}$ norm

- shifting bound on the $\mathscr{H}_{2}$ norm

is considered.

Remark 1. Despite in this paper the problem of design using shifting specification is considered for the case of LPV systems, the proposed method is useful for LTI systems too. In this case, a vector $\theta_{p}(t)$, exogenous with respect to the system to be controlled, is introduced, and used to schedule the controller, such that, even though the plant to be controlled is LTI, the overall system is LPV and the mathematical reasoning developed hereafter can be applied. The reason to do so is that in this way the performance of the closed-loop system can be varied in time according to some criterium, e.g. energetic issues. The introduction of an exogenous $\theta_{p}(t)$ can also be done in the case of LPV systems, when it is desired to vary the performances according to criteria that are not connected with the intrinsec varying parameters of the LPV system. 


\subsection{Shifting pole placement for LPV systems}

Following the definition of poles for LPV systems provided by [44], let us define, for a given value $\theta_{p}^{*}$ of $\theta_{p}(t), \sigma\left(\theta_{p}^{*}\right)$ as the set of all the poles of the LTI systems obtained from (16)-(18) by freezing $\theta_{S}(t)$ and $\theta_{r}(t)$ to all their possible values $\theta_{s}^{*}$ and $\theta_{r}^{*}$ in $\Theta_{s}$ and $\Theta_{r}$, respectively. The problem considered hereafter, and denoted as shifting pole placement problem in the following, consists in designing the controller (27) such that $\sigma\left(\theta_{p}(t)\right) \in \mathscr{D}\left(\theta_{p}(t)\right) \forall \theta_{p} \in \Theta_{p}$, where $\mathscr{D}\left(\theta_{p}(t)\right)$ is an LMI region whose characteristic function depends on $\theta_{p}(t)$, as follows:

$$
\begin{aligned}
& f_{\mathscr{D}}\left(z, \theta_{p}(t)\right)=\alpha\left(\theta_{p}(t)\right)+z \beta\left(\theta_{p}(t)\right)+\bar{z} \beta\left(\theta_{p}(t)\right)^{T} \\
& =\left[\alpha_{k l}\left(\theta_{p}(t)\right)+\beta_{k l}\left(\theta_{p}(t)\right) z+\beta_{l k}\left(\theta_{p}(t)\right) \bar{z}\right]_{1 \leq k, l \leq m}
\end{aligned}
$$

In this case, the shifting pole placement problem is solved if there exist a symmetric positive definite matrix $X=X^{T}>0$ and an LPV state-feedback gain $K\left(\theta_{s}(t), \theta_{p}(t)\right)$ such that:

$$
\begin{aligned}
& M_{\mathscr{D}\left(\theta_{p}(t)\right)}\left(A(\theta(t))+B_{u}(\theta(t)) K\left(\theta_{s}(t), \theta_{p}(t)\right), X\right) \\
& \quad=\alpha\left(\theta_{p}(t)\right) \otimes X+\beta\left(\theta_{p}(t)\right) \otimes\left[\left(A(\theta(t))+B_{u}(\theta(t)) K\left(\theta_{s}(t), \theta_{p}(t)\right)\right) X\right] \\
& \quad+\beta\left(\theta_{p}(t)\right)^{T} \otimes\left[\left(A(\theta(t))+B_{u}(\theta(t)) K\left(\theta_{s}(t), \theta_{p}(t)\right)\right) X\right]^{T}=\left\{\alpha_{k l}\left(\theta_{p}(t)\right)\right. \\
& \quad+\beta_{k l}\left(\theta_{p}(t)\right)\left[A(\theta(t))+B_{u}(\theta(t)) K\left(\theta_{s}(t), \theta_{p}(t)\right)\right] X \\
& \left.\quad+\beta_{l k}\left(\theta_{p}(t)\right) X\left[A(\theta(t))+B_{u}(\theta(t)) K\left(\theta_{s}(t), \theta_{p}(t)\right)\right]^{T}\right\}_{1 \leq k, l \leq m}^{<0}<
\end{aligned}
$$

holds for each $\theta \in \Theta$.

It can be shown that the definition of shifting pole placement allows to vary in time the transient performance of the closed-loop system, i.e. its decay or growth rate. This is demonstrated by the following theorem, that is based on the results provided by [6] and [45] for the case of the classical regional pole placement.

Theorem 2. Consider a parameter-varying LMI region $\mathscr{D}(\theta(t))$ with characteristic function:

$$
f_{\mathscr{D}(\theta(t))}(z, \theta(t))=\alpha(\theta(t))+z \beta(\theta(t))+\bar{z} \beta(\theta(t))^{T}
$$

and assume that the continuous-time dynamical system:

$$
\dot{x}(t)=A(\theta(t)) x(t)
$$

is quadratically $\mathscr{D}(\theta(t))$-stable, i.e. there exists $X=X^{T}>0$ such that:

$M_{\mathscr{D}(\theta(t))}(A(\theta(t)), X)=\alpha(\theta(t)) \otimes X+\beta(\theta(t)) \otimes X A(\theta(t))+\beta(\theta(t))^{T} \otimes A(\theta(t))^{T} X<0$ 
for all $\theta \in \Theta$. Then, the quadratic function $V(x(t))=x(t)^{T} X x(t)$ satisfies, for all $x(t) \neq 0$ :

$$
\frac{\dot{V}(x(t))}{2 V(x(t))} \in \mathscr{D}(\theta(t)) \cap \mathbb{R}
$$

Proof: The proof resembles the ones provided by [6] and [45]. Multiplying (32) left and right by $I \otimes x(t)^{T}$ and $I \otimes x(t)$ respectively, the following is obtained for all $x(t) \neq 0$ :

$$
\alpha(\theta(t)) \otimes x(t)^{T} X x(t)+\beta(\theta(t)) \otimes x(t)^{T} X A(\theta(t)) x(t)+\beta(\theta(t))^{T} \otimes x(t)^{T} A(\theta(t))^{T} X x(t)<0
$$

Then, recalling that $\dot{V}(x(t)) / 2=x(t)^{T} X A(\theta(t)) x(t)=x(t)^{T} A(\theta(t)) X x(t)$, and dividing by $V(x(t))$, the following is obtained:

$$
\alpha(\theta(t)) \otimes 1+\beta(\theta(t)) \otimes \frac{\dot{V}(x(t))}{2 V(x(t))}+\beta(\theta(t))^{T} \otimes \frac{\dot{V}(x(t))}{2 V(x(t))}<0
$$

which, by definition of $\mathscr{D}(\theta(t))$, ensures:

$$
\frac{\dot{V}(x(t))}{2 V(x(t))} \in \mathscr{D}(\theta(t))
$$

that completes the proof.

Looking at Theorem 2, it can be seen that using a shifting pole placement specification, it is possible to modify online the constraint on the minimum decay rate (if $\mathscr{D}(\theta(t))$ is contained in the left half plane), or the maximum possible growth rate of the Lyapunov function used to assess the $\mathscr{D}(\theta(t))$-stability. From a practical point of view, it is possible to vary online other transient performance, e.g. the damping characteristics.

However, condition (29) cannot be used for the controller design, since it imposes an infinite number of constraints. This difficulty can be alleviated under the assumptions that $\beta\left(\theta_{p}(t)\right)$ is a constant matrix, that $B_{u}(\theta(t))$ only depends on $\theta_{r}(t)$, and that the matrices $\alpha\left(\theta_{p}(t)\right), A(\theta(t)), B_{u}\left(\theta_{r}(t)\right)$ and $K\left(\theta_{s}(t), \theta_{p}(t)\right)$ vary polytopically as follows:

$$
\begin{gathered}
\alpha\left(\theta_{p}(t)\right)=\sum_{h=1}^{P} \pi_{h}\left(\theta_{p}(t)\right) \alpha_{h} \\
A(\theta(t))=\sum_{i=1}^{S} s_{i}\left(\theta_{S}(t)\right) \sum_{j=1}^{R} r_{j}\left(\theta_{r}(t)\right) \sum_{h=1}^{P} \pi_{h}\left(\theta_{p}(t)\right) A_{i j h}
\end{gathered}
$$




$$
\begin{gathered}
B_{u}\left(\theta_{r}(t)\right)=\sum_{j=1}^{R} r_{j}\left(\theta_{r}(t)\right) B_{u, j} \\
K\left(\theta_{s}(t), \theta_{p}(t)\right)=\sum_{i=1}^{S} s_{i}\left(\theta_{s}(t)\right) \sum_{h=1}^{P} \pi_{h}\left(\theta_{p}(t)\right) K_{i h}
\end{gathered}
$$

Looking at the examples of LMI regions given in Section 2, it can be seen that the assumption of constant $\beta$ matrix corresponds to fixing the shape of the shifting LMI region. On the other hand, in cases where the assumption that $B_{u}(\theta(t))$ only depends on $\theta_{r}(t)$ introduces too much conservativeness, it is possible to relax this assumption by filtering the inputs as proposed in [46])

Then, it is possible to reduce (29) to a finite number of matrix inequalities, by rewriting it at the $S \cdot R \cdot P$ vertices of the polytope $\Theta$, as stated in the following theorem:

Theorem 3. Let $\mathscr{D}\left(\theta_{p}(t)\right)$ be an LMI region whose characteristic function depends on the scheduling parameter $\theta_{p}(t)$ as in (28), and assume that a symmetric positive definite matrix $X=X^{T}>0$ and $S \cdot P$ matrices $K_{11}, \ldots, K_{i h}, \ldots, K_{S P}$ have been found such that:

$$
\left\{\alpha_{k l, h}+\beta_{k l}\left(A_{i j h}+B_{u, j} K_{i h}\right) X+\beta_{l k} X\left(A_{i j h}+B_{u, j} K_{i h}\right)^{T}\right\}_{1 \leq k, l \leq m}^{<0}
$$

for each $i=1, \ldots, S, j=1, \ldots, R$ and $h=1, \ldots, P$.

Then, given $\left[\begin{array}{lll}\theta_{S}(t)^{T} & \theta_{r}(t)^{T} & \theta_{p}(t)^{T}\end{array}\right]^{T} \in \Theta$ with polytopic decompositions (22)-(24), condition (29) results satisfied for each $\theta$ in $\Theta$. Hence, the controller (27) places the closed-loop poles of (16) in $\mathscr{D}\left(\theta_{p}(t)\right)$.

Proof: Due to a basic property of matrices [47], any linear combination of (41) with non-negative coefficients is negative definite. Hence, using the linear combinations brought by (22)-(24) leads to:

$$
\sum_{i=1}^{S} s_{i}\left(\theta_{s}(t)\right) \sum_{j=1}^{R} r_{j}\left(\theta_{r}(t)\right) \sum_{h=1}^{P} \pi_{h}\left(\theta_{p}(t)\right)\left\{\alpha_{k l, h}+\beta_{k l}\left(A_{i j h}+B_{u, j} K_{i h}\right) X+\beta_{l k} X\left(A_{i j h}+B_{u, j} K_{i h}\right)^{T}\right\} \underset{1 \leq k, l \leq m}{<0}
$$

Bringing inside the brackets $\sum_{i=1}^{S} s_{i}, \sum_{j=1}^{R} r_{j}$ and $\sum_{h=1}^{P} \pi_{h}$, and taking into account the assumption that $\beta\left(\theta_{p}(t)\right)$ is constant, and (37)-(40), the following result is obtained:

$$
\begin{aligned}
& \left\{\alpha_{k l}\left(\theta_{p}(t)\right)+\beta_{k l}\left[A(\theta(t))+B_{u}\left(\theta_{r}(t)\right) K\left(\theta_{s}(t), \theta_{p}(t)\right)\right] X\right. \\
& \left.\quad+\beta_{l k} X\left[A(\theta(t))+B_{u}\left(\theta_{r}(t)\right) K\left(\theta_{s}(t), \theta_{p}(t)\right)\right]^{T}\right\} \\
& \quad \begin{array}{c}
<\leq k, l \leq m \\
1 \leq 0
\end{array}
\end{aligned}
$$

that proves the theorem. 
Notice that (41) are bilinear matrix inequalities (BMIs) due to the products $K_{i h} X$, and thus the problem to be solved is not convex. However, using the auxiliary variables $L_{i h}=K_{i h} X$, convexity is restored and the BMIs are transformed into LMIs, that can be easily solved. Then, once a feasible solution $\left\{X^{*}, L_{i h}^{*}, i=1, \ldots, N, h=1, \ldots, P\right\}$ has been found, the state-feedback gains can be obtained as $K_{i h}^{*}=L_{i h}^{*}\left(X^{*}\right)^{-1}$.

Remark 2. As a consequence of Theorem 3, if the LMI region $\mathscr{D}\left(\theta_{p}(t)\right)$ is inside the left half plane for all the possible values of $\theta_{p}$ in $\Theta_{p}$, (41) is a sufficient condition for the closed-loop stability of (16) with the controller (27). In cases where the intersection between $\mathscr{D}\left(\theta_{p}(t)\right)$ and the right half plane is not empty, the closed-loop stability could be analyzed using the theory of LPV systems with brief instabilities [48]. However, such analysis goes beyond the goal of this paper, and will be addressed in future work.

Remark 3. Notice that the total number of variables that need to be found is $S P+$ 1. The LMIs (41), together with the condition of positive definiteness of the matrix $X$, can be rewritten as a single block-diagonal LMI whose size is $n_{x}(1+m S R P)$.

\subsection{Shifting bound on the $\mathscr{H}_{\infty}$ norm for LPV systems}

The problem considered hereafter consists in designing the controller (27) for the system (16)-(17) such that the closed-loop transfer function from $w$ to $z_{\infty}$ satisfies the shifting bound on the $\mathscr{H}_{\infty}$ norm specification:

$$
\left\|T_{z_{\infty} w}(s, \theta(t))\right\|_{\infty}<\gamma\left(\theta_{p}(t)\right)
$$

As a consequence of the BRL (10), this problem is solved if there exist a positive definite symmetric matrix $X_{\infty}=X_{\infty}^{T}>0$ and an LPV state-feedback gain $K\left(\theta_{s}(t), \theta_{p}(t)\right)$ such that:

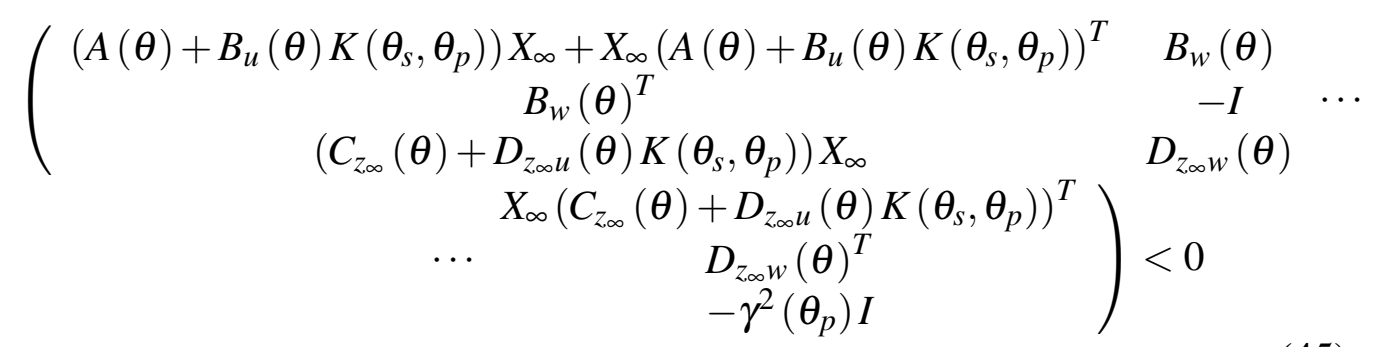


holds for each $\theta \in \Theta^{1}$.

In this case, the assumptions that $B_{u}(\theta(t))$ and $D_{z_{\infty} u}(\theta(t))$ only depend on $\theta_{r}(t)$ (also in this case, the use of a filter [46] can relax this assumption), and that the matrices and the shifting bound vary polytopically as in (40) and as follows:

$$
\begin{gathered}
\left(\begin{array}{c}
A(\theta(t)) \\
B_{w}(\theta(t)) \\
C_{z_{\infty}}(\theta(t)) \\
D_{z_{\infty} w}(\theta(t))
\end{array}\right)=\sum_{i=1}^{S} s_{i}\left(\theta_{s}(t)\right) \sum_{j=1}^{R} r_{j}\left(\theta_{r}(t)\right) \sum_{h=1}^{P} \pi_{h}\left(\theta_{p}(t)\right)\left(\begin{array}{c}
A_{i j h} \\
B_{w, i j h} \\
C_{z_{\infty}, i j h} \\
D_{z_{\infty} w, i j h}
\end{array}\right) \\
\left(\begin{array}{c}
B_{u}\left(\theta_{r}(t)\right) \\
D_{z_{\infty} u}\left(\theta_{r}(t)\right)
\end{array}\right)=\sum_{j=1}^{R} r_{j}\left(\theta_{r}(t)\right)\left(\begin{array}{c}
B_{u, j} \\
D_{z_{\infty} u, j}
\end{array}\right) \\
\gamma^{2}\left(\theta_{p}(t)\right)=\sum_{h=1}^{P} \pi_{h}\left(\theta_{p}(t)\right) \gamma_{h}^{2}
\end{gathered}
$$

are needed in order to reduce the constraint (45) to a finite number of matrix inequalities, by rewriting it at the $S \cdot R \cdot P$ vertices of the polytope $\Theta$, as stated in the following theorem:

Theorem 4. Let $\gamma\left(\theta_{p}(t)\right)$ be a desired bound on the $\mathscr{H}_{\infty}$ norm that depends on the scheduling parameter $\theta_{p}(t)$ and assume that a symmetric positive definite matrix $X_{\infty}=X_{\infty}^{T}>0$ and $S \cdot P$ matrices $K_{11}, \ldots, K_{i h}, \ldots, K_{S P}$ have been found such that:

$$
\left(\begin{array}{ccc}
\left(A_{i j h}+B_{u, j} K_{i h}\right) X_{\infty}+X_{\infty}\left(A_{i j h}+B_{u, j} K_{i h}\right)^{T} & B_{w, i j h} & X_{\infty}\left(C_{z_{\infty}, i j h}+D_{z_{\infty} u, j} K_{i h}\right)^{T} \\
B_{w, i j h}^{T} & -I & D_{z_{\infty} w_{i}, i j h} \\
\left(C_{z_{\infty}, i j h}+D_{z_{\infty} u, j} K_{i h}\right) X_{\infty} & D_{z_{\infty} w, i j h} & -\gamma_{h}^{2} I
\end{array}\right)<0
$$

for each $i=1, \ldots, S, j=1, \ldots, R$ and $h=1, \ldots, P$.

Then, given $\left[\begin{array}{lll}\theta_{s}(t)^{T} & \theta_{r}(t)^{T} & \theta_{p}(t)^{T}\end{array}\right]^{T} \in \Theta$ with polytopic decompositions (22)-(24), the condition (45) results satisfied. Hence, the controller (27) satisfies the shifting bound on the $\mathscr{H}_{\infty}$ norm specification (44).

Proof: The proof resembles the one of Theorem 3, and is omitted.

Also in this case, although the problem to be solved is not convex, convexity is restored using the auxiliary variables $L_{i h}=K_{i h} X$.

\footnotetext{
${ }^{1}$ The dependence of $\theta$ on $t$ has been omitted due to lack of space.
} 
Remark 4. Applying the Schur complement [49] to the upper-left element of (45), the Lyapunov stability condition is obtained. Hence, the stability is guaranteed and there is no need to prove it separately.

Remark 5. Notice that the total number of variables that need to be found is $S P+1$. The LMIs (49), together with the condition of positive definiteness of $X_{\infty}$, can be rewritten as a single block-diagonal LMI whose size is $n_{x}(1+S R P)+\left(n_{w}+\right.$ $\left.n_{z_{\infty}}\right) S R P$.

\subsection{Shifting bound on the $\mathscr{H}_{2}$ norm for LPV systems}

In this case, it is wished to design the controller (27) for the system (16), (18) such that the closed-loop transfer function from $w$ to $z_{2}$ satisfies the shifting bound on the $\mathscr{H}_{2}$ norm specification:

$$
\left\|T_{z_{2} w}(s, \theta(t))\right\|<v\left(\theta_{p}(t)\right)
$$

As a consequence of (13), the constraint (50) is equivalent to the existence of a positive definite symmetric matrix $X_{2}=X_{2}^{T}$, a symmetric matrix $Y(\theta(t))=$ $Y(\theta(t))^{T}$ and an LPV state-feedback gain $K\left(\theta_{s}(t), \theta_{p}(t)\right)$ such that:

$$
\begin{gathered}
\operatorname{trace}(Y(\theta(t)))<v^{2}\left(\theta_{p}(t)\right) \\
Y(\theta(t)) \\
\left(\begin{array}{c}
\cdots \\
X_{2}\left(C_{z_{2}}(\theta(t))+D_{z_{2} u}(\theta(t)) K\left(\theta_{s}(t), \theta_{p}(t)\right)\right)^{T} \\
\left(C_{z_{2}}(\theta(t))+D_{z_{2} u}(\theta(t)) K\left(\theta_{s}(t), \theta_{p}(t)\right)\right) X_{2} \\
X_{2}
\end{array}\right)>0 \\
\left(A(\theta(t))+B_{u}(\theta(t)) K\left(\theta_{s}(t), \theta_{p}(t)\right)\right) X_{2}+X_{2}\left(A(\theta(t))+B_{u}(\theta(t)) K\left(\theta_{s}(t), \theta_{p}(t)\right)\right)^{T} \\
+B_{w}(\theta(t)) B_{w}(\theta(t))^{T}<0
\end{gathered}
$$

hold for each $\theta \in \Theta$.

In this case, the assumptions that $B_{u}(\theta(t))$ and $D_{z_{2} u}(\theta(t))$ only depend on $\theta_{r}(t)$, and that the matrices and the shifting bound vary polytopically, as in (40) and as follows:

$$
\left(\begin{array}{c}
A(\theta(t)) \\
C_{z_{2}}(\theta(t)) \\
Y(\theta(t)) \\
B_{w}(\theta(t))
\end{array}\right)=\sum_{i=1}^{S} s_{i}\left(\theta_{s}(t)\right) \sum_{j=1}^{R} r_{j}\left(\theta_{r}(t)\right) \sum_{h=1}^{P} \pi_{h}\left(\theta_{p}(t)\right)\left(\begin{array}{c}
A_{i j h} \\
C_{z_{2}, i j h} \\
Y_{i j h} \\
B_{w, i j h}
\end{array}\right)
$$




$$
\begin{gathered}
\left(\begin{array}{c}
B_{u}\left(\theta_{r}(t)\right) \\
D_{z_{2} u}\left(\theta_{r}(t)\right)
\end{array}\right)=\sum_{j=1}^{R} r_{j}(\theta(t))\left(\begin{array}{c}
B_{u, j} \\
D_{z_{2} u, j}
\end{array}\right) \\
v^{2}\left(\theta_{p}(t)\right)=\sum_{h=1}^{P} \pi_{h}\left(\theta_{p}(t)\right) v_{h}^{2}
\end{gathered}
$$

are needed in order to reduce the constraint (51)-(53) to a finite number of matrix inequalities, by rewriting them at the $S \cdot R \cdot P$ vertices of the polytope $\Theta$, as stated in the following theorem:

Theorem 5. Let $v\left(\theta_{p}(t)\right)$ be a desired bound on the $\mathscr{H}_{2}$ norm that depends on the scheduling parameter $\theta_{p}(t)$ and assume that a symmetric positive definite matrix $X_{2}=X_{2}^{T}>0, S \cdot P$ matrices $K_{11}, \ldots, K_{i h}, \ldots, K_{S P}$ and $S \cdot R \cdot P$ matrices $Y_{111}, \ldots, Y_{i j h}, \ldots, Y_{S R P}$ have been found such that:

$$
\begin{gathered}
\operatorname{trace}\left(Y_{i j h}\right)<v_{h}^{2} \\
\left(\begin{array}{cc}
Y_{i j h} & \left(C_{z_{2}, i j h}+D_{z_{2} u, j} K_{i h}\right) X_{2} \\
X_{2}\left(C_{z_{2}, i j h}+D_{z_{2} u, j} K_{i h}\right)^{T} & X_{2}
\end{array}\right)>0 \\
\left(A_{i j h}+B_{u, j} K_{i h}\right) X_{2}+X_{2}\left(A_{i j h}+B_{u, j} K_{i h}\right)^{T}+B_{w, i j h} B_{w, i j h}^{T}<0
\end{gathered}
$$

for each $i=1, \ldots, S, j=1, \ldots, R$ and $h=1, \ldots, P$.

Then, given $\left[\begin{array}{lll}\theta_{s}(t)^{T} & \theta_{r}(t)^{T} & \theta_{p}(t)^{T}\end{array}\right]^{T} \in \Theta$ with polytopic decompositions (22)-(24), conditions (51)-(53) result satisfied. Hence, the controller (27) satisfies the shifting bound on the $\mathscr{H}_{2}$ norm specification (50).

Proof: The proof resembles that of Theorem 3, and is omitted.

Also in this case, although the problem to be solved is not convex, convexity is restored using the auxiliary variables $L_{i h}=K_{i h} X$.

Remark 6. In this case, the stability is guaranteed by the positive semidefiniteness of the matrix $B_{w}(\theta(t)) B_{w}(\theta(t))^{T}$ in (53) for each $\theta \in \Theta$ [50].

Remark 7. Notice that the total number of variables that need to be found is $S P(R+1)+1$. The LMIs (57)-(59), together with the condition of positive definiteness of $X_{2}$, can be rewritten as a single block-diagonal LMI of size $n_{x}(1+$ $2 S R P)+\left(1+n_{z_{2}}\right) S R P$. 


\section{Simulation Example}

The example proposed in this paper is an LPV mathematical system that illustrates some of the features of the proposed approach for the design of parameterscheduled state-feedback controllers using shifting specifications, and refers to a continuous-time LPV system with a structure as in (16)-(17) (the $\mathscr{H}_{2}$ norm will not be considered, because of its similarity with the $\mathscr{H}_{\infty}$ norm), and with matrices defined as follows:

$$
\begin{gathered}
A(\theta(t))=\left(\begin{array}{cccc}
0 & 1 & 0 & 0 \\
-\theta_{p}(t) & -\theta_{s}(t) & \theta_{p}(t) & 0 \\
0 & 0 & 0 & 1 \\
\theta_{p}(t) & 0 & -\theta_{p}(t) & -\theta_{r}(t)
\end{array}\right) \\
B_{u}\left(\theta_{r}(t)\right)=\left(\begin{array}{cc}
0 & 0 \\
\theta_{r}(t) & 0 \\
0 & 0 \\
0 & 1
\end{array}\right) \\
B_{w}=\left(\begin{array}{c}
1 \\
0 \\
0 \\
0
\end{array}\right) \\
C_{z_{\infty}}(\theta(t))=\left(\begin{array}{ccc}
\theta_{p}(t) & 0 & 0 \\
D_{z_{\infty} u}=D_{z_{\infty} w}=0
\end{array}\right)
\end{gathered}
$$

where $\theta_{s} \in[2,3], \theta_{r} \in[2,3]$ and $\theta_{p} \in[1,2]$ (in this example, the subscripts $s, r, p$ are used following the notation explained in Section 3). Notice that the assumption made in Section 3 about the matrix $B_{u}$ depending only on the subset of varying parameters $\theta_{r}(t)$ is verified.

\subsection{Shifting pole placement}

In this case, the controller gain $K\left(\theta_{s}(t), \theta_{p}(t)\right)$ is designed such that the closedloop poles of the LPV system are placed in a disk of radius $r\left(\theta_{p}(t)\right)$ and center $\left(-q\left(\theta_{p}(t)\right), 0\right)$, described by the characteristic function:

$$
f_{\mathscr{D}}\left(z, \theta_{p}(t)\right)=\left(\begin{array}{cc}
-r\left(\theta_{p}(t)\right) & q\left(\theta_{p}(t)\right)+z \\
q\left(\theta_{p}(t)\right)+\bar{z} & -r\left(\theta_{p}(t)\right)
\end{array}\right)
$$

with $r\left(\theta_{p}(t)\right)$ and $q\left(\theta_{p}(t)\right)$ defined as: 
Table 1: Varying parameters and polytopic vertices

\begin{tabular}{|c|c|c|c|c|c|}
\hline$\theta_{s}$ & $\theta_{r}$ & $\theta_{p}$ & $A_{i j h}$ & $K_{i h}$ & $B_{u, j}$ \\
\hline 2 & 2 & 1 & $A_{111}$ & $K_{11}$ & $B_{u, 1}$ \\
\hline 2 & 2 & 2 & $A_{112}$ & $K_{12}$ & $B_{u, 1}$ \\
\hline 2 & 3 & 1 & $A_{121}$ & $K_{11}$ & $B_{u, 2}$ \\
\hline 2 & 3 & 2 & $A_{122}$ & $K_{12}$ & $B_{u, 2}$ \\
\hline 3 & 2 & 1 & $A_{211}$ & $K_{21}$ & $B_{u, 1}$ \\
\hline 3 & 2 & 2 & $A_{212}$ & $K_{22}$ & $B_{u, 1}$ \\
\hline 3 & 3 & 1 & $A_{221}$ & $K_{21}$ & $B_{u, 2}$ \\
\hline 3 & 3 & 2 & $A_{222}$ & $K_{22}$ & $B_{u, 2}$ \\
\hline
\end{tabular}

$$
\left\{\begin{array}{l}
r\left(\theta_{p}(t)\right)=1+\theta_{p}(t) \\
q\left(\theta_{p}(t)\right)=-1+3 \theta_{p}(t)
\end{array}\right.
$$

The design is done using (41), that in this case involves nine matrix inequalities in the variables $X, K_{11}, K_{12}, K_{21}, K_{22}$ (see Table 1 ):

$$
\left\{\begin{array}{cc}
-2 X & 2 X+A_{111} X+B_{u, 1} K_{11} X \\
2 X+X A_{111}^{T}+X K_{11}^{T} B_{u, 1}^{T} & -2 X \\
-3 X & 5 X+A_{112} X+B_{u, 1} K_{12} X
\end{array}\right)<0
$$

that are solved using the YALMIP toolbox [51] with SeDuMi solver [52], with the 
following solution:

$$
\begin{gathered}
X=\left(\begin{array}{cccc}
9.5331 & -23.2373 & -0.0810 & 0.4356 \\
-23.2373 & 73.5810 & -0.0192 & -1.1634 \\
-0.0810 & -0.0192 & 8.8115 & -21.8937 \\
0.4356 & -1.1634 & -21.8937 & 71.7764
\end{array}\right) \\
K_{11}=\left(\begin{array}{cccc}
-1.5979 & -0.9678 & -0.4216 & 0.0135 \\
-0.9590 & 0.0185 & -4.0906 & -1.9989
\end{array}\right) \\
K_{12}=\left(\begin{array}{cccc}
-4.0292 & -2.1305 & -0.8277 & -0.0080 \\
-1.8067 & 0.0519 & -10.7803 & -5.0865
\end{array}\right) \\
K_{21}=\left(\begin{array}{cccc}
-1.5949 & -0.5824 & -0.4214 & -0.0138 \\
-0.9574 & 0.0182 & -4.0908 & -1.9989
\end{array}\right) \\
K_{22}=\left(\begin{array}{cccc}
-3.9800 & -1.7225 & -0.8233 & -0.0081 \\
-1.8151 & 0.0433 & -10.7816 & -5.0870
\end{array}\right)
\end{gathered}
$$

Table 2 lists the eigenvalues of the vertex closed-loop matrices $A_{i j h}+B_{u, j} K_{i h}$. Also, Fig. 1 shows how the closed-loop poles shift according to different values of the scheduling parameter $\theta_{p}$, proving that the shifting pole placement specification is correctly satisfied. In particular, it can be seen that in contrast with the classical regional pole placement approach, where a region is selected and all the poles of the closed-loop system are forced to be in such region, the shifting pole placement approach allows to select different regions for different values of the scheduling parameter $\theta_{p}$. According to Theorem 2, the decay rate of the Lyapunov function varies with the value of $\theta_{p}$. This fact has the direct consequence that also the transient dynamics varies with $\theta_{p}$. In fact, by taking a look at the dominant poles of the vertex systems in Table 2 , one can see that the range of dominant poles for $\theta_{p}=1$ (index $h=1$ ) is $[-1.4256,-1.9655]$, while when $\theta_{p}=2($ index $h=2)$ the range of the dominant poles is $[-3.1499,-2.1652]$.

The effect of the shifting pole placement specification on the transient dynamics of the closed-loop system can be effectively seen by taking a look at the free responses of the state variables, shown in Figs. 2-5. These free responses have been obtained starting from the initial state $x(0)=\left[\begin{array}{llll}1 & 0 & 1 & 0\end{array}\right]^{T}$ in four different cases, three of which with constant values of the scheduling parameter $\theta_{p}(t)\left(\theta_{p}=1, \theta_{p}=1.5\right.$ and $\theta_{p}=2$, corresponding to blue solid, purple dashed and red dotted line, respectively), and one with a varying scheduling parameter 
Table 2: Closed-loop eigenvalues of the matrices $A_{i j h}+B_{u, j} K_{i h}$

\begin{tabular}{|c|c|c|c|c|}
\hline$A_{i j h}+B_{u, j} K_{i h}$ & Eig. 1 & Eig. 2 & Eig. 3 & Eig. 4 \\
\hline$A_{111}+B_{u, 1} K_{11}$ & $-1.9655+0.5672 \mathrm{j}$ & $-1.9655-0.5672 \mathrm{j}$ & $-2.0018+1.0464 \mathrm{j}$ & $-2.0018-1.0464 \mathrm{j}$ \\
\hline$A_{112}+B_{u, 1} K_{12}$ & $-3.1499+0.4752 \mathrm{j}$ & $-3.1499-0.4752 \mathrm{j}$ & $-3.5239+0.4937 \mathrm{j}$ & $-3.5239-0.4937 \mathrm{j}$ \\
\hline$A_{121}+B_{u, 2} K_{11}$ & -1.4256 & -1.9845 & -2.9160 & -3.5763 \\
\hline$A_{122}+B_{u, 2} K_{12}$ & -2.1702 & -2.3052 & -5.9139 & -6.0888 \\
\hline$A_{211}+B_{u, 1} K_{21}$ & -1.6971 & $-2.0010+1.0459 \mathrm{j}$ & $-2.0010-1.0459 \mathrm{j}$ & -2.4644 \\
\hline$A_{212}+B_{u, 1} K_{22}$ & -2.5525 & $-3.5451+0.4991 \mathrm{j}$ & $-3.5451-0.4991 \mathrm{j}$ & -3.8892 \\
\hline$A_{221}+B_{u, 2} K_{21}$ & -1.4256 & $-2.3724+0.3890 \mathrm{j}$ & $-2.3724-0.3890 \mathrm{j}$ & -3.5757 \\
\hline$A_{222}+B_{u, 2} K_{22}$ & -2.1652 & -2.4186 & -5.7294 & -5.9412 \\
\hline
\end{tabular}

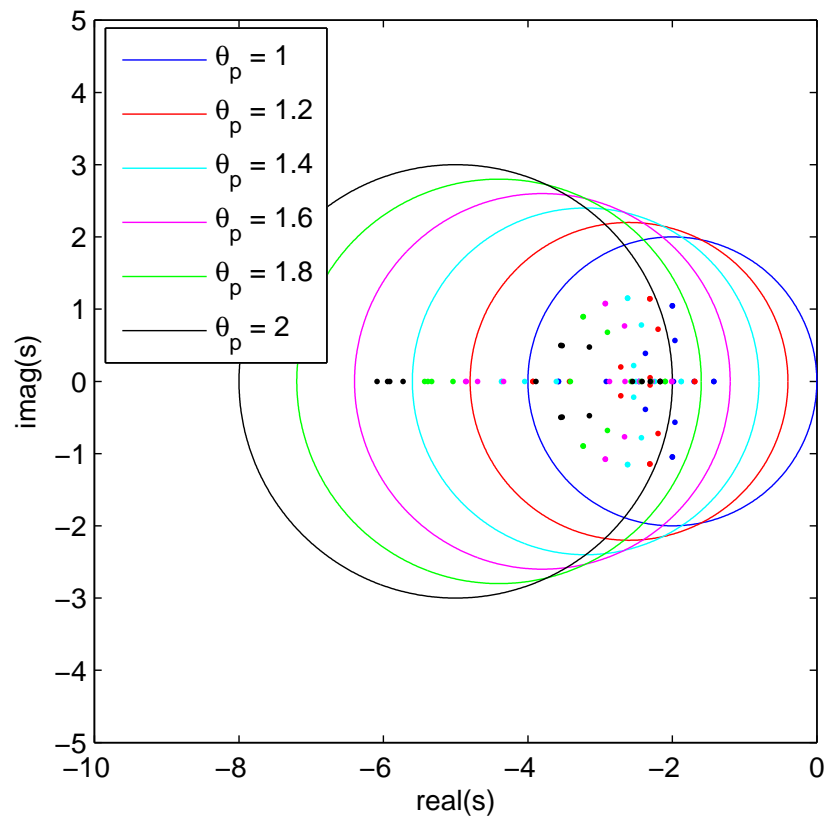

Figure 1: Shifting pole placement for the LPV mathematical system. 


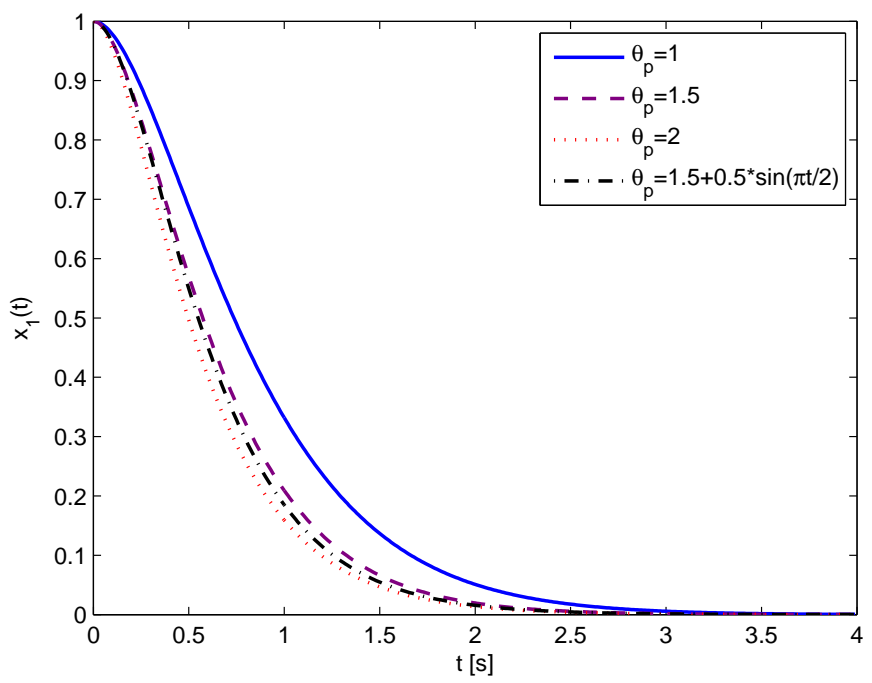

Figure 2: Free response of $x_{1}(t)$ (closed-loop).

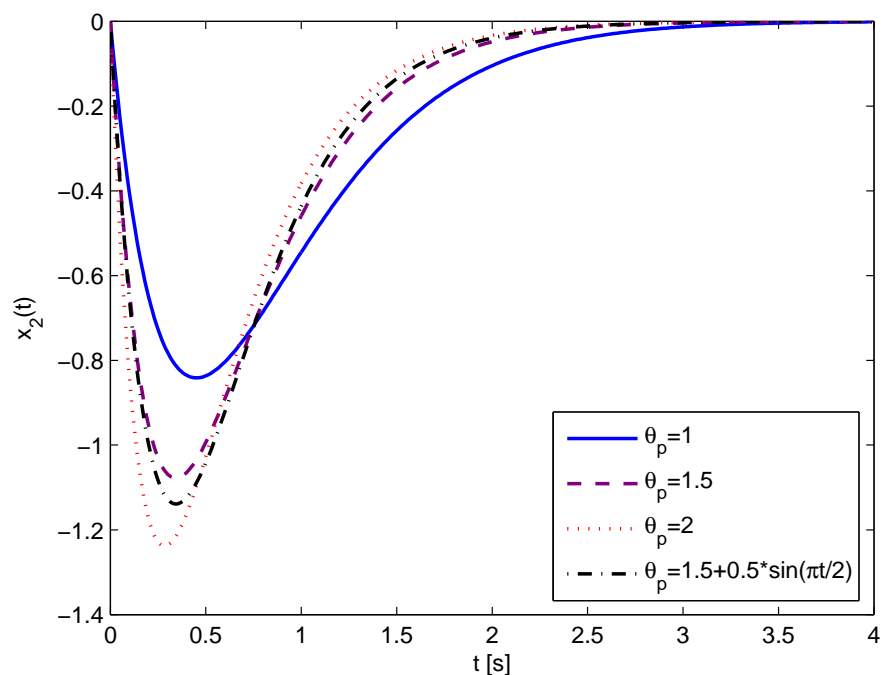

Figure 3: Free response of $x_{2}(t)$ (closed-loop). 


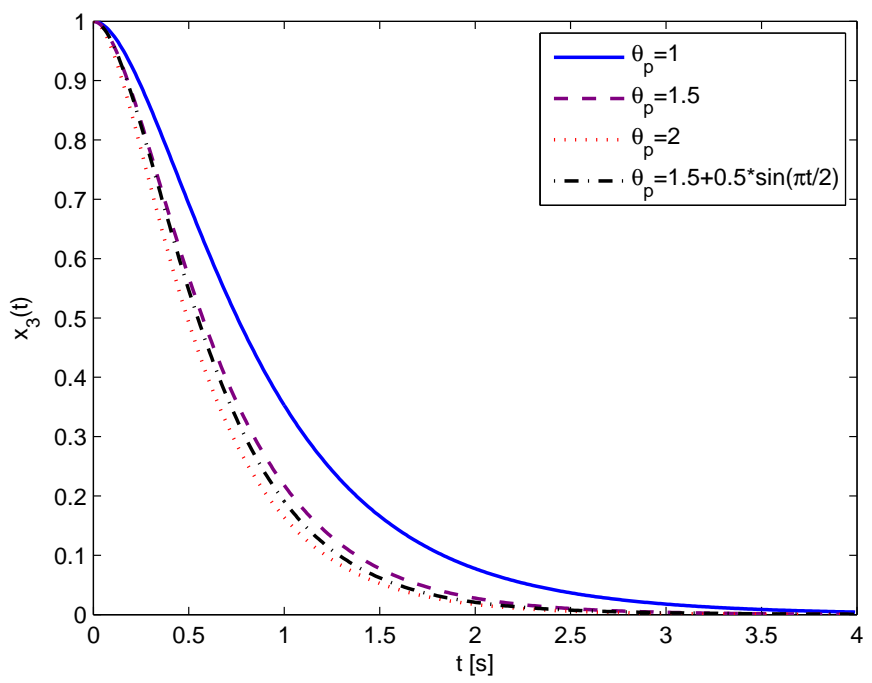

Figure 4: Free response of $x_{3}(t)$ (closed-loop).

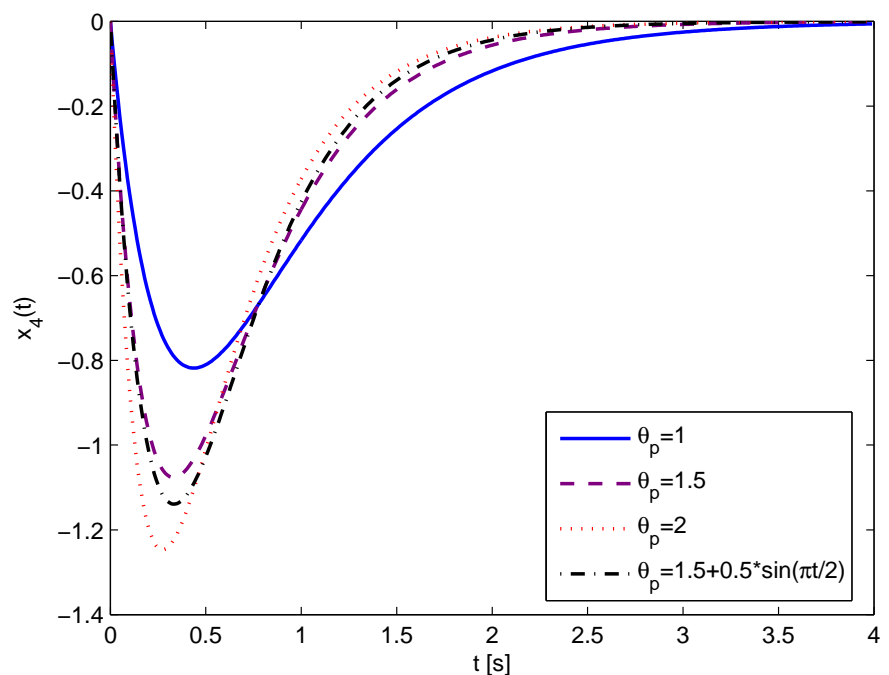

Figure 5: Free response of $x_{4}(t)$ (closed-loop). 
$\theta_{p}(t)=1.5+0.5 \sin (\pi t / 2)$ (corresponding to black dash-dotted line). The remaining scheduling parameters have been chosen as $\theta_{s}(t)=2.5+0.5 \cos t$ and $\theta_{r}(t)=2.5+0.5 \sin t$.

It can be seen from the figures that the closed-loop system behaves as expected: a big value of $\theta_{p}$ corresponds to faster dynamics of the closed-loop system. In the fourth case, that is, with a time-varying $\theta_{p}$, the dynamics of the closedloop system around $t=0 \mathrm{~s}$ is the same as that of the closed-loop system scheduled by the constant $\theta_{p}=1.5$. As the time increases, so does the value of $\theta_{p}$ and the system gets faster until $t=1 s$ when $\theta_{p}$ begins to decrease and the trend reverses, with the system getting slower. However, this last effect and the increasing of speed from time $t=3 \mathrm{~s}$ are not appreciable because the steady-state has almost been reached. The input signals $u_{1}$ and $u_{2}$ are shown in Fig. 6 and Fig. 7. It can be seen that the bigger is $\theta_{p}$, the bigger are the control signals, and vice versa. This is consistent with the fact that strong control actions are required to make the controlled system faster.

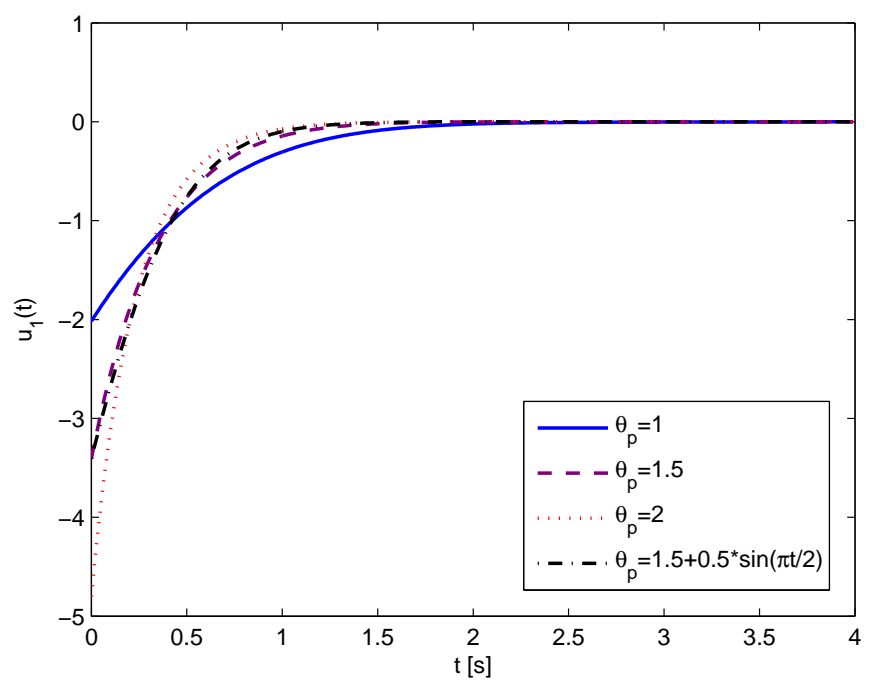

Figure 6: Input signal $u_{1}(t)$.

\subsection{Shifting bound on the $\mathscr{H}_{\infty}$ norm}

In this case, the controller gain $K\left(\theta_{s}(t), \theta_{p}(t)\right)$ has been designed such that the transfer function from $w$ to $z_{\infty}$ satisfies the following desired bound on the $\mathscr{H}_{\infty}$ norm: 


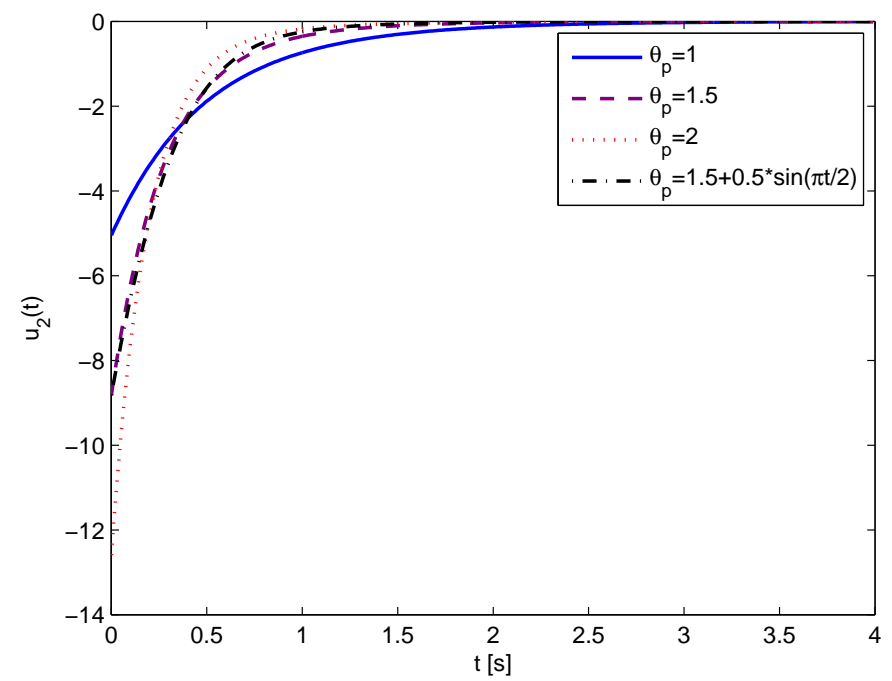

Figure 7: Input signal $u_{2}(t)$.

$$
\gamma\left(\theta_{p}(t)\right)=\sqrt{0.01+0.24\left(\theta_{p}(t)-1\right)}
$$

Notice that the particular structure of the shifting bound (68) allows to obtain (48) through a simple algebraic manipulation, as follows:

$$
\gamma^{2}\left(\theta_{p}(t)\right)=\pi_{1}\left(\theta_{p}(t)\right) \gamma_{1}^{2}+\pi_{2}\left(\theta_{p}(t)\right) \gamma_{2}^{2}
$$

with $\gamma_{1}^{2}=0.01, \gamma_{2}^{2}=0.25$ and:

$$
\pi_{1}\left(\theta_{p}(t)\right)=2-\theta_{p}(t) \quad \pi_{2}\left(\theta_{p}(t)\right)=\theta_{p}(t)-1
$$

The design is done using (49), that in this case is made up by nine LMIs in the variables $X_{\infty}, K_{11}, K_{12}, K_{21}, K_{22}$, that are solved using the YALMIP toolbox [51] with SeDuMi solver [52], providing the following solution:

$$
\begin{gathered}
X_{\infty}=\left(\begin{array}{cccc}
0.0562 & -0.7985 & 0.0120 & 0.0042 \\
-0.7985 & 11.9183 & -0.2890 & -0.0497 \\
0.0120 & -0.2890 & 12.1721 & -6.0386 \\
0.0042 & -0.0497 & -6.0386 & 6.1784
\end{array}\right) \\
K_{11}=\left(\begin{array}{cccc}
-1588.9 & -107.2 & -2.5 & -2.2 \\
155.7 & 11.0 & -1.0 & -0.9
\end{array}\right)
\end{gathered}
$$




$$
\begin{gathered}
K_{12}=\left(\begin{array}{cccc}
-12.8429 & -5.2293 & -2.3686 & -2.4228 \\
4.8911 & 3.8083 & -0.5264 & -1.4244
\end{array}\right) \\
K_{21}=\left(\begin{array}{cccc}
-1570.2 & -105.6 & -2.4 & -2.2 \\
154.6 & 10.9 & -1.0 & -0.9
\end{array}\right) \\
K_{22}=\left(\begin{array}{cccc}
-12.6380 & -4.7831 & -2.3922 & -2.5209 \\
5.7649 & 4.2455 & -0.4902 & -1.3834
\end{array}\right)
\end{gathered}
$$

It can be seen from the figures that the closed-loop system behaves as expected. The shifting bound on the $\mathscr{H}_{\infty}$ norm specification results satisfied for each possible value of the scheduling parameter $\theta_{p}$, as depicted in Fig. 8. It can be seen that the relevant feature of the shifting approach with respect to the classical $\mathscr{H}_{\infty}$ design, is that it allows to specify different bounds for the $\mathscr{H}_{\infty}$ norm corresponding to different values of $\theta_{p}(t)$, thus allowing to vary online the exogenous input rejection characteristics. The effect of the proposed specification on the closed-loop system can be seen graphically by plotting the Bode plot of $T_{z_{\infty} w}$ for different values of the parameter $\theta_{p}$ (see Fig. 9). In particular, it can be seen that the higher is the value of $\theta_{p}$, the higher is the peak of the magnitude of $T_{z_{\infty} w}$, a result that is consistent with the definition of $\mathscr{H}_{\infty}$ norm and the proposed shifting counterpart. Finally, to conclude this analysis, let us consider a sinusoidal exogenous input $w=\sin (t)$, and let us analyse the response of the closed-loop system to this input starting from zero initial conditions. As in the shifting pole placement example previously presented, the simulations are performed with $\theta_{s}(t)=2.5+0.5 \cos t$ and $\theta_{r}(t)=2.5+0.5 \sin t$, and different values of $\theta_{p}(t)$ have been considered for comparison purpose. The obtained results are plotted in Fig. 10. As expected, to a small value of $\theta_{p}$ (in this case, $\theta_{p}=1$ ) corresponds a stronger rejection of the exogenous input (blue solid line). By increasing $\theta_{p}$, e.g. to values of $\theta_{p}=1.5$ (purple dashed line) and $\theta_{p}=2$ (red dotted line), the rejection performance of the control system decreases. When considering a varying $\theta_{p}(t)=1.5+0.5 \sin (\pi t / 2)$, the rejection characteristics vary in time: at the beginning of the simulation, when $\theta_{p}(t)$ is approximately 1.5 , the response of the system with the varying $\theta_{p}$ (black dash-dotted line) equals the one with a constant $\theta_{p}=1.5$ (purple dashed line). As the time increases, so does $\theta_{p}(t)$, and the gain of the transfer function from $w$ to $z_{\infty}$ increases. Hence, the effect of the sinusoidal signal on $z_{\infty}$ becomes stronger and stronger until time $t=1 \mathrm{~s}$, when the varying parameter $\theta_{p}(t)$ begins to decrease again, and the trend reverses. 


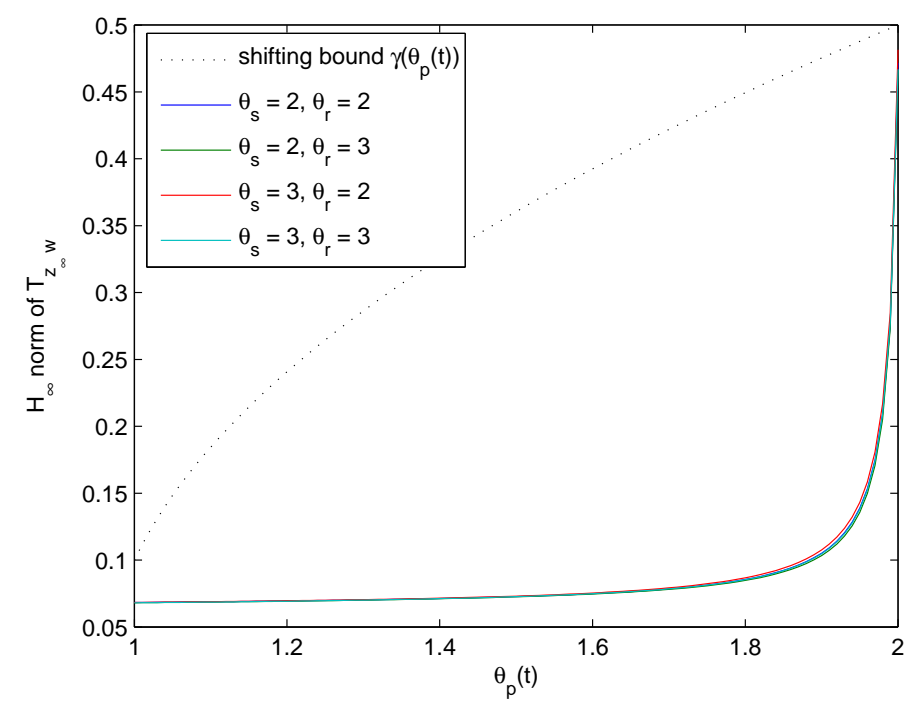

Figure 8: Shifting bound on the $\mathscr{H}_{\infty}$ norm.

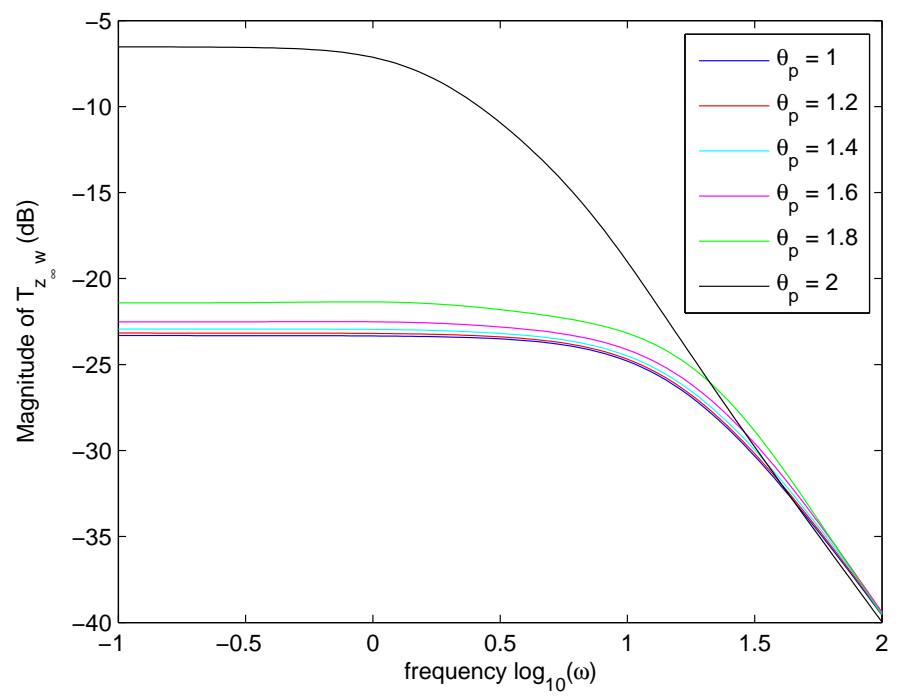

Figure 9: Variation of the Bode plot according to changes in $\theta_{p}$, obtained for $\theta_{s}=\theta_{r}=2$. 


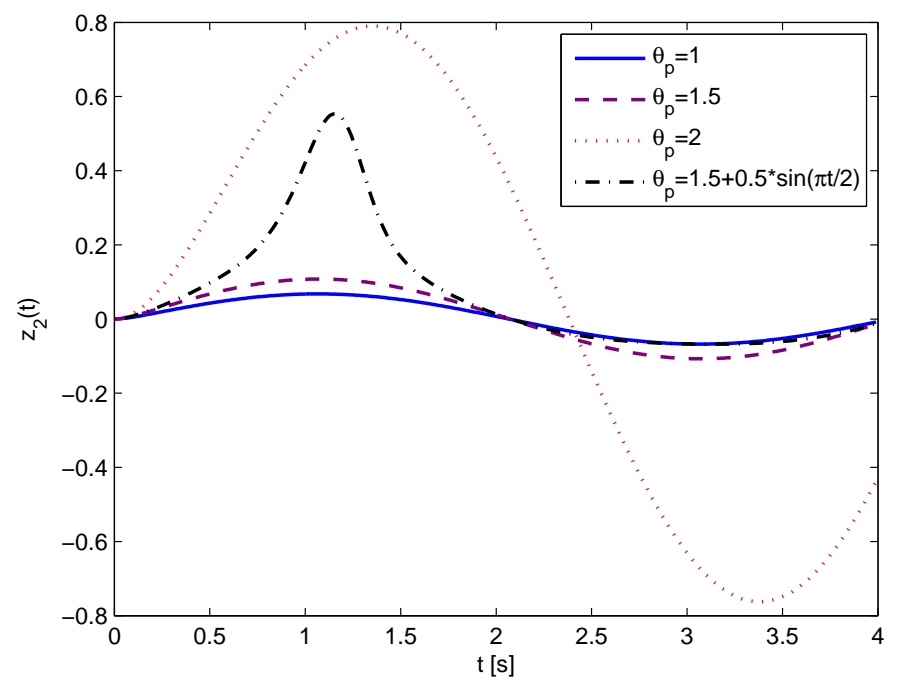

Figure 10: Response of the closed-loop system to an exogenous input $w=\sin (t)$.

\section{Conclusions}

In this paper, the problem of designing a parameter-scheduled state-feedback controller that satisfies a new kind of specifications, referred to as shifting specifications, has been investigated. In particular, the regional pole placement, the bound on the $\mathscr{H}_{\infty}$ norm and the bound on the $\mathscr{H}_{2}$ norm have been extended in a shifting sense, introducing the shifting pole placement, the shifting bound on the $\mathscr{H}_{\infty}$ norm and the shifting bound on the $\mathscr{H}_{2}$ norm specifications. The problem has been analyzed in the LPV case, and the solution has been obtained using a single Lyapunov function and expressed as a system of LMIs for which a feasible solution should be found. The results obtained with an LPV mathematical example have demonstrated the effectiveness and some characteristics of the proposed approach. In particular, in contrast with the classical regional pole placement and $\mathscr{H}_{\infty} / \mathscr{H}_{2}$ control approaches, the design using shifting specifications allows to select different performances for different values of the scheduling parameters $\theta_{p}$, thus allowing to vary online the control system performance.

Future work will focus on applying the proposed approach to all those situations where some performance degradation could be desirable, e.g. control of systems with saturation non-linearities and graceful performance degradation in fault tolerant control. Moreover, since the use of a single Lyapunov function is 
potentially conservative, the application of parameter-dependent Lyapunov functions will be investigated too.

\section{Acknowledgements}

This work has been funded by the Spanish MINECO through the project CYCYT SHERECS (ref. DPI2011-26243), by the European Commission through contract i-Sense (ref. FP7-ICT-2009-6-270428), by AGAUR through the contracts FI-DGR 2013 (ref. 2013FIB00218) and FI-DGR 2014 (ref. 2014FI_B1 00172) and by the DGR of Generalitat de Catalunya (SAC group Ref. 2014/SGR/374).

[1] S. Boyd, L. E. Ghaoui, E. Feron, V. Balakrishnan, Linear matrix inequalities in system and control theory, SIAM Studies in Applied Mathematics, 1994.

[2] M. Chilali, P. Gahinet, $H_{\infty}$ design with pole placement constraints: an LMI approach, IEEE Transactions on Automatic Control 41 (1996) 358-367.

[3] J. Ackermann, Sampled-data control systems, Berlin, Germany: SpringerVerlag, 1985.

[4] S. Gutman, E. I. Jury, A general theory for matrix root clustering in subregions of the complex plane, IEEE Transactions on Automatic Control AC-26 (1981) 853-863.

[5] P. Gahinet, P. Apkarian, M. Chilali, Affine parameter-dependent Lyapunov functions and real parametric uncertainty, IEEE Transactions on Automatic Control 41 (1996) 436-442.

[6] M. Chilali, P. Gahinet, P. Apkarian, Robust pole placement in LMI regions, IEEE Transactions on Automatic Control 44 (1999) 2257-2270.

[7] R. M. Palhares, P. L. D. Peres, Robust $H_{\infty}$ filter design with pole constraints for discrete-time systems, Journal of the Franklin Institute 337 (2000) 713723.

[8] V. J. S. Leite, P. L. D. Peres, An improved LMI condition for robust $\mathscr{D}$ stability of uncertain polytopic systems, IEEE Transactions on Automatic Control 48 (2003) 500-504.

[9] W. Assawinchaichote, S. K. Nguang, $H_{\infty}$ filtering for fuzzy singularly perturbed systems with pole placement constraints: an LMI approach, IEEE Transactions on Signal Processing 52 (2004) 1659-1667. 
[10] N. Maamri, O. Bachelier, D. Mehdi, Pole placement in a union of regions with prespecified subregion allocation, Mathematics and Computers in Simulation 72 (2006) 38-46.

[11] S. K. Nguang, P. Shi, Robust $H_{\infty}$ output feedback control design for fuzzy dynamic systems with quadratic $\mathscr{D}$ stability constrants: an LMI approach, Information Sciences 176 (2006) 2161-2191.

[12] F. Yang, M. Gani, D. Henrion, Fixed-order robust $H_{\infty}$ controller design with regional pole assignment, IEEE Transactions on Automatic Control 52 (2007) 1959-1963.

[13] D. H. Lee, J. B. Park, Y. H. Joo, K. C. Lin, Lifted versions of robust $\mathscr{D}$ stability and $\mathscr{D}$-stabilisation conditions for uncertain polytopic linear systems, IET Control Theory and Applications 6 (2012) 24-36.

[14] G. Zames, Feedback and optimal sensitivity: model reference transformations, multiplicative seminorms, and approximate inverses, IEEE Transactions on Automatic Control AC-26 (1981) 301-320.

[15] K. Glover, J. Doyle, State-space formulae for all stabilizing controllers that satisfy an $\mathscr{H}_{\infty}$ norm bound and relations to risk sensitivity, Systems and Control Letters 11 (1988) 167-172.

[16] J. C. Doyle, K. Glover, P. P. Khargonekar, B. A. Francis, State-space solutions to standard $\mathscr{H}_{2}$ and $\mathscr{H}_{\infty}$ control problems, IEEE Transactions on Automatic Control AC-34 (1989) 831-847.

[17] T. Iwasaki, R. E. Skelton, All controllers for the general $\mathscr{H}_{\infty}$ control problem: LMI existence conditions and state space formulas, Automatica 30 (1994) 1307-1317.

[18] P. Gahinet, P. Apkarian, A linear matrix inequality approach to $\mathscr{H}_{\infty}$ control, International Journal of Robust and Nonlinear Control 4 (1994) 421-448.

[19] J. Yu, A. Sideris, $\mathscr{H}_{\infty}$ control with parametric Lyapunov functions, Systems and Control Letters 30 (1997) 57-69.

[20] X. Chen, K. Zhou, Multiobjective $\mathscr{H}_{2} \backslash \mathscr{H}_{\infty}$ control design, SIAM Journal on Control and Optimization 40 (2001) 628-660. 
[21] M. C. D. Oliveira, J. C. Geromel, J. Bernussou, Extended $\mathscr{H}_{2}$ and $\mathscr{H}_{\infty}$ norm characterizations and controller parametrizations for discrete-time systems, International Journal of Control 75 (2002) 666-679.

[22] E. N. Gonçalves, R. M. Palhares, R. H. C. Takahashi, Improved optimisation approach to the robust $\mathscr{H}_{2} \backslash \mathscr{H}_{\infty}$ control problem for linear systems, IEE Proceedings on Control Theory and Applications 152 (2005) 171-176.

[23] D. Rotondo, F. Nejjari, V. Puig, A shifting pole placement approach for the design of parameter-scheduled state-feedback controllers, in: Proceedings of the 12th European Control Conference, Zürich, Switzerland, pp. 18291834, 2013.

[24] F. Wu, K. M. Grigoriadis, A. Packard, Anti-windup controller design using linear parameter-varying control methods, International Journal of Control 73 (2000) 1104-1114.

[25] Y. Zhang, J. Jiang, D. Theilliol, Incorporating performance degradation in fault tolerant control system design with multiple actuator failures, International Journal of Control, Automation, and Systems 6 (2008) 327-338.

[26] A. Khelassi, D. Theilliol, P. Weber, J.-C. Ponsart, Fault-tolerant control design with respect to actuator health degradation: An LMI approach, in: Proceedings of the International Conference on Control Applications (CCA), 2011.

[27] P. Apkarian, P. Gahinet, A convex characterization of gain-scheduled $\mathscr{H}_{\infty}$ controllers, IEEE Transactions on Automatic Control 40 (1995) 853-864.

[28] D. Peaucelle, D. Arzelier, O. Bachelier, J. Bernussou, A new robust $\mathscr{D}$ stability condition for real convex polytopic uncertainty, Systems and Control Letters 40 (2000) 21-30.

[29] L. Xie, S. Shishkin, M. Fu, Piecewise Lyapunov functions for robust stability of linear time-varying systems, Systems and Control Letters 31 (1997) 165171.

[30] R. Murray-Smith, T. A. Johansen, Multiple model approaches to nonlinear modeling and control, Taylor and Francis, London, 1997. 
[31] T. Takagi, M. Sugeno, Fuzzy identification of systems and its applications to modeling and control, IEEE Transactions on Systems, Man, and Cybernetics SMC-15 (1985) 116-132.

[32] R. Babuška, Fuzzy modeling for control, Kluwer, Boston, MA, 1998.

[33] K. Tanaka, H. O. Wang, Fuzzy control systems design and analysis: a linear matrix inequality approach, John Wiley and Sons, Inc., 2001.

[34] P. M. Mäkilä, P. Viljamaa, Convex parametric design, gain scheduling, and fuzzy computing, Technical Report, Tampere, Tampere University of Technology, Institute of Automation and Control, 2002.

[35] P. Bergsten, R. Palm, D. Driankov, Observers for Takagi-Sugeno fuzzy systems, IEEE Transactions on Systems, Man, and Cybernetics - Part B: Cybernetics 32 (2002) 114-121.

[36] Q. Rong, G. W. Irwin, LMI-based control design for discrete polytopic LPV systems, in: Proceedings of the 6th European Control Conference, 2003.

[37] A. Graham, Kronecker product and matrix calculus with applications, New York: Wiley, 1981.

[38] B. A. Francis, A course in $\mathscr{H}_{\infty}$ control theory, Berlin: Springer-Verlag, 1987.

[39] C. Scherer, The Riccati inequality and state-space $\mathscr{H}_{\infty}$-optimal control, Ph.D. thesis, Univ. Wirzburg, Germany, 1990.

[40] C. W. Scherer, P. Gahinet, M. Chilali, Multi-objective output feedback control via LMI optimization, IEEE Transactions on Automatic Control 42 (1997) 896-911.

[41] J. Shamma, J. Cloutier, A linear parameter-varying approach to gain scheduled missile autopilot design, in: Proceedings of the 11th American Control Conference, pp. 1317-1321, 1992.

[42] D. Rotondo, F. Nejjari, V. Puig, Robust state-feedback control of uncertain LPV systems: an LMI-based approach, Journal of the Franklin Institute 351 (2014) 2781-2803.

[43] J. Pfeifle, V. Pilaud, F. Santos, Polytopality and cartesian products of graphs, Israel Journal of Mathematics 192 (2012) 121-141. 
[44] A. Ghersin, R. Sanchez-Peña, LPV control of a 6-DOF vehicle, IEEE Transactions on Control Systems Technology 10 (2002) 883-887.

[45] W. Assawinchaichote, S. K. Nguang, P. Shi, E.-K. Boukas, $H_{\infty}$ fuzzy statefeedback control design for nonlinear systems with $\mathscr{D}$-stability constraints: an LMI approach, Mathematics and Computers in Simulation (MATCOM) 78 (2008) 514-531.

[46] P. Apkarian, P. Gahinet, G. Becker, Self-scheduled $\mathscr{H}_{\infty}$ control of linear parameter-varying systems: a design example, Automatica 9 (1995) 12511261.

[47] R. A. Horn, C. R. Johnson, Matrix analysis, Cambridge University Press, 1990.

[48] J. M. Hespanha, O. A. Yakimenko, I. I. Kaminer, A. M. Pascoal, Linear parametrically varying systems with brief instabilities: an application to vision/inertial navigation, IEEE Transactions on Aerospace and Electronic Systems 40 (2004) 899-902.

[49] J. Schur, Über Potenzreihen, die im Innern des Einheitskreises beschränkt sind, Journal für die reine und angewandte Mathematik 147 (1917) 205-232.

[50] H. Abdi, L. J. Williams, Matrix Algebra, in: N. Salkind (Ed.), Encyclopedia of Research Design, Thousand Oaks, CA: Sage, 2010, pp. 1-32.

[51] J. Löfberg, YALMIP: a toolbox for modeling and optimization in MATLAB, in: Proceedings of the CACSD Conference, 2004.

[52] J. F. Sturm, Using SeDuMi 1.02, a MATLAB toolbox for optimization over symmetric cones, Optimization methods and software 11-12 (1999) 625653. 